\title{
Chromosomal Interchanges in Barley ${ }^{1}$
}

\author{
C. R. Burnham, F. H. White and R. Livers ${ }^{2}$
}

Receired Jamuary 10, 1954

Studies thus far of interchange heterozygotes in maize indicate little or no directed segregation of the chromosomes in the ring (5), pollen and ovule abortion below 50 per cent not being observed except in cases where at least one of the interchanged pieces may be so short that its deficiency does not cause spore abortion (3). Interchanges in barley, Hordeum vulgare, and in Triticum monococcum, (the latter furnished by the late Dr. Luther Smith) show lower degrees of sterility, suggesting directed segregation as one possible explanation. Those in monococcum are not being continued, but our limited counts have shown that the pollen abortion of plants carrying a ring of four chromosomes ranged from 11 to $16 \%$ in different interchange lines. This may be high enough to identify ring-carrying plants by pollen examination. These values appear to be higher than the five to $10 \%$ for the translocations in T. monococcum reported by Thompson and Thompson (22). Yamashita (26) reported a range of 7 to $17 \%$ pollen abortion and an average of nine per cent for five rings-of-four in Aegilopoides monococcum and two in T. monococcum. He reported a mean of $83 \%$ of zigzag configurations at metaphase $I$.

This is a preliminary report on a group of interchanges in barley which has been the subject of several thesis problems in our laboratory $(8,25)$ and in others $(21,24)$. Earlier reports have been presented(7, 7a, 25).

In 1943 seed of Mars, a smooth-awned variety adapted to Minnesota, was X-rayed (20,000 r-units) by Dr. L. J. Stadler, and planted in the greenhouse by Dr. M. T. Henderson. The heads from these plants were harvested individually, separated into two groups, those apparently normal and those showing partial sterility. The seed from each head was space-planted in separate rows in the field. Although planted for another study, at the time of pollen shedding, three or four plants from each row were examined for pollen abortion. For this purpose, three to six florets from one head were removed, placed in a vial of 70 per cent alcohol, and after a few hours the pollen was examined under the microscope in $\mathrm{KI}-\mathrm{I} 2$ solution. By doing this as soon as the plants started shedding pollen, it was still possible to obtain usable sporocyte samples from tillers on most of the plants showing pollen abortion. The acetocarmine smear technique was used for the cytolo-

1 Published as Scientifie Journal Series Paper No. 3092 of the Minnesota Agricultural Experiment Station, Department of Agronomy and Plant Geneties, St. Paul, Minnesota. This is the paper referred to by Smith (20a) as "in Press", Agronomy Journal).

2 University of Minnesota; Tobacco Division, Central Experimental Farm, Ottawa Ontario: and Plains Substation, Clovis, New Mexico. 
gical study. There is a tendency for the cytoplasm to become too dark, but it can be destained by allowing 45 per cent acetic acid to run under the cover slip, and then heating nearly to boiling. To make the slides permanent, a modification of the technique described by McClintock is satisfactory. The steps are as follows: step 1, remove the temporary seal and soak off the cover slip in 10 per cent acetic acid; then pass the cover slip and slide through: step 2, equal parts of glacial acetic acid, and absolute alcohol: step 3,9 parts of absolute alcohol; 1 part glacial acetic: steps 4 and 5, two changes of absolute alcohol, then apply balsam to the slide and add the cover slip. The two changes of absolute alcohol at the end appear to be essential; possibly the third step might be omitted.

\section{Results}

From the partially sterile heads, there were 143 rows with at least three plants sampled. Of these, 48 had at least one plant with an estimated pollen abortion of 20 or more percent and eight had at least one with roughly ten per cent. Of the former group of 48 rows, 27 had plants with a ring or a chain of four chromosomes plus five pairs indicating a translocation heterozygote, one had two separate rings of four chromosomes each plus three pairs (Fig. 1); and 13 had only seven pairs (the sporocytes for plants in seven lines were too old). Of the eight lines with lower sterility, the five that were examined had seven pairs of chromosomes.

In the group of 27 rows from normal-appearing parent heads, one had 20 or more per cent pollen abortion and had a ring of four chromosomes and one had less than ten per cent (no cytology). This group, although small, had a much lower frequency of lines showing partially sterile pollen than did the group from partially sterile heads, 7 vs. $39 \%$ of the rows.

One line with about 20 per cent pollen abortion had from one to six "long chromosomes" and occasional cells with univalents at metaphase I, strikingly similar to published figures of a similar character in Matthiola, (1, 15). This character in barley behaves as a simple recessive in inheritance, $(4,17)$ and is linked with naked $(n)$ in linkage group III (17).

The other partially sterile lines have been studied by Das (8). Bridge plus fragment formation was found at anaphase $I$ in one line, suggesting the presence of an inversion.

In all but two cases, each line was established from one partially sterile plant in the original head-row in the field to avoid the possibility that different interchanges might be segregating in a row. This original plant was the one examined cytologically.

Since these lines are being studied extensively, both genetically and cytologically, at this and several other laboratories, the interchange lines are listed in Table 1, including counts on pollen and ovule abortion, and the workers studying them further. Interchanges $A$ and $B$, first reported by 
Smith (20), A being studied later by Joachim (14), are included. The culture or c-numbers are being used here as identification numbers until they can be identified in terms of their chromosome morphology or the linkage groups. Pollen abortion in plants with one ring of four chromosomes ranged in different lines from 14 (c 1462-2) to 58 per cent (c 1438-1,) the average being $28.8 \%$. It was 51 per cent in the plants with two separate rings of four (c1376-1). Ovule abortion on these same plants was 39, 92, and 67 per cent respectively. The data on ovule abortion are based on seed-set counts of two heads on the original partially sterile plant, and may not necessarily be the true degree of sterility. There appears to be additional ovule sterility in certain lines which may be due to causes other than the interchange. In several of the lines, a chain or string of four chromosomes was frequent. In one line (c 1184), 7 pairs were observed usually with only an occasional chain or a ring of four chromosomes.

The ultimate purpose of the studies has been to identify the chromosomes involved in the interchanges by intercrossing them, and by means of linkage studies with genetic markers to determine the linkage group carried by each of the chromosomes. The studies were expected to determine if the seven linkage groups as established by linkage studies (Robertson et al $(18,19)$ ) do represent the expected seven independent groups corresponding to the seven chromosome pairs.

As more information on breakage points and linkages becomes available they may be used to supplement the genetic markers for use in linkage tests; and as tools for studying special problems, e.g. in the study of more complex characters, chromosome segregation, and possibly in breeding programs.

The plan for further study has been to intercross the interchange lines, and examine the $F_{1}$ plants carrying both interchanges; a ring or group of six indicating a chromosome common to the two; two separate rings of four indicating none in common. Since only one-fourth of the progeny from the intercross of two heterozygous lines should carry both interchanges, it was decided to isolate lines homozygous for each interchange as the first step. In intercrosses between such lines or in crosses with linkage testers, all the $F_{1}$ plants can be used. For the intercrosses it is highly desirable to have at least one parent homozygous. Theoretically from self-pollination, half the normal plants in the progeny of a plant heterozygous for an interchange should be homozygous for that interchange. To identify them, the same general method has been used as described in maize (2), i.e. self the plants with normal pollen and also cross them on to a standard normal line. If the normal being tested were homozygous for the interchange, all the $F_{1}$ plants should be partially sterile.

A stock segregating male sterility was selected as the standard normal line to facilitate crossing, the male sterile plants being used as females without emasculation. Open pollinated seed of male sterile plants which would seg- 
regate in a 1:1 ratio was kindly furnished by C. A. Suneson. We are now using the progeny of plants which are heterozygous $m s$, since the openpollinated $m s$ plants seldom set any seed here. For the first trial, three normal plants from each partially sterile line were tested. These tests have been continued, and at the present time 28 of the 34 lines whose cytology shows a ring have been established in homozygous condition. These are indicated by an asterisk $\left({ }^{*}\right)$ after the identification numbers in Table 1 . One was identified by Dr. Wilson Foote, one by Dr. R. W Woodward, and the others by the present writers. No difficulty has been encountered in establishing them, except possibly for $\mathrm{C} 951$, from which eight normals have been tested without identifying the interchange homozygote. In the tests establishing the homozygous lines, 36 plants have proved to be standard normals and 45 were homozygous for the interchange, close to the $1: 1$ ratio expected. One for the " B" interchange and one for "A" have been established also. Seed of a group of five homozygous lines being studied by White for his $\mathrm{Ph} . \mathrm{D}$. thesis was also furnished to the other workers to permit correlation of all the studies. Plans for cooperation in publication are expected to materialize, for the later work. Hanson and Kramer $(11,13)$ have reported linkage data for two of them, and Hanson (12) on two more.

Based on cytological observations of intercrosses between the interchanges a tester set has been selected, by means of which the chromosomes involved in any new interchange should be identifiable.

A scheme for selecting a usable tester set has been found to be helpful after intercross results are available for several interchanges. If the haploid chromosomes are divided into groups of three $(a, b, c),(d, e, f)$, etc.; for any group of three chromosomes, two interchange testers are sufficient to identify any translocation involving one or more of them. Three different sets of two each for each group of three chromosomes are possible and equally satisfactory. For example, for the $a, b, c$ group, the three sets of two each are : $\mathrm{a}-b$ and $b-\mathrm{c}, \mathrm{a}-c$ and $\mathrm{b}-c$, or $a-\mathrm{b}$ and $a-\mathrm{c}$. It will be noted that in each set of two, one chromosome is involved in both tester interchanges. For the six chromosomes included in the two groups of three each, four testers are sufficient*

The seven chromosomes have been given the letters $a$ to $g$ temporarily until the linkage group for each has been established without question or until their identification in relation to Tjio and Hagberg's I to VII designa-

* This scheme has been useful in selecting complete tester sets for maize. In our studies of maize interchanges, a tester set of six interchanges has been used to identify nine of the ten chromosomes. Chromosome 6 is not involved in any of those in the tester set, but since it is the only one associated with the nucleolus any interchange involving that chromosome can be identified easily by the association of the ring with the nucleolus. In barley two chromosomes appear to be associated with the nucleolus and also diakinesis stages are more difficult to find and interpret than in maize, hence the need for a more complete tester set in barley. 
Table 1. List of interchanges. All but A and B were isolated from X-rayed seed of Mars barley. Included are the available pollen and ovule abortion data, and the workers who are carrying on studies subsequent to their isolation.

Identification number
Translocation
A*
B*
c 951
e $1025^{*}$
c 1184
c $1310^{*}$
c $1315^{*}$
c 1316
c $1317^{*}$
c 1333
c $1336^{*}$
c $1340^{*}$
c $1343^{*}$
c $1346^{*}$
c $1358^{*} t t$
c $1363^{*}$
c $1365^{*}$
c 1376
$\quad " 1$
c $1377^{*}$
ring

Lines with no eytology

c 1428

c 1440

c 1460

c 1467

c 1468

c 1478

c 1490

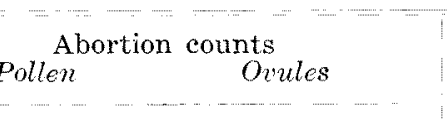

Workers

-
-
30,0
21.3
43.1
37.7
25.9
26.2
23.2
40.5
19.2
20.3

20.3

21.9

51.3

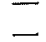

35.8

31.2

27.6

31.3

25.7

30.9

33.4

22.0

28.5

58.0

28.9

36.8

14.4

18.3

21.5

24.0

estimated pollen

abortion

25

30

20

30

20

25

30
48.5

62.0

43.5

55.7

27.9

60.6

43.2

25.7

70.2

55.2

38.0

25.0

66.9

-

36.8

65.3

31.3

31.2

27.2

34.4

20.6

36.7

30.7

92.2

30.9

34.9

38.9

39.7

40.4

52.3

counted ovule

abortion

28.8

31.5

6.9

38.5

44.4

43.5
1

1

1

3

1

4

1

1

4

1

1

2

2

1

1

$t 1=$ F. H. White, H. A. MeLennan, R. Livers, Univ. of Minnesota, St. Paul, Minn. $2=$ R. W. Woodward, and M. Waddoups, Utah Agric. College, Logan. Utah.

$3=$ H. H. Kramer and W. D. Hanson, Purdue Agr. Exp. Sta. Lafayette, Ind. $4=0$.J. Webster and $O$. Smith, College of Agr. Univ. of Nebr., Lincoln, Nebr. tt The interchange tester set for identifying new interchanges.

$*$ =homozygous interchange stock established. 
tions based on chromosome morphology has been completed (23). The tester set originally included the following four interchanges : c 1385 (a-b), c 1358 (b-d), c 1432 (c-e), and c 1420 (e-f). An intercross between the first two or between the last two shows a ring of six chromosomes at diakinesis or metaphase of meiosis, and intercrosses between any of the others result in two separate rings of four chromosomes. Results from intercrosses between 16 interchanges (including these testers) made by White also served to check and establish the designations assigned to these four testers. These four serve to identify all but two of the 21 different possible combinations involving non-homologues. With this tester set, a b-g interchange cannot be distinguished from $\mathrm{a}-\mathrm{d}$; and $\mathrm{e}-\mathrm{g}$ cannot be distinguished from $c-f$. The other combinations involving $g$ are identified by elimination. Since the $g$ chromosome appears to involve the large satellite, those involving that chromosome should be associated with the nucleolus, determinable by cytological examination at diakinesis. Since diakinesis stages are not always easily found, a fifth tester, c 1405 (c-d) has been added to the set. This will distinguish between the interchanges not completely identifiable with the other four testers. These five homozygous interchanges are now being used as a standard identification set. For three of the interchanges, the Suneson male sterile ( $m s$ ) gene has been introduced to facilitate crossing. It is being introduced into the other two also. These are built up by the following scheme: 1. cross the homozygous interchange on an $m s m s$ plant; 2 . in the $F_{2}$ select the male-sterile segregates and cross them again with the homozygous interchange stock; 3 . examine the pollen of the resulting plants. Any plants that are normal should be the desired homozygous interchange which will carry $m s$ and segregate $m s m s$ plants in its progeny. The $m s m s$ plant in step 2 from which they came would have been either heterozygous or homozygous for the interchange. The method may be useful in introducing other genes into interchange stocks.

The cytological results from the intercrosses of these testers are shown in Table 2. Also included are the results of intercrosses between the tester set and four other interchanges, illustrating their use.

Off-type plants (shorter and later than normal, or with different leaf shape), were observed in the progeny of plants heterozygous for certain of the interchanges. Examination of sporocytes from 12 such plants showed four of them to be trisomic, usually a chain of five chromosomes in place of the normal ring of four. From their progeny, it should be possible to select stocks of primary trisomics, i.e. without the interchange.

The interchanges listed in Table 1 were all identified first by the presence of pollen abortion. Since plants having rings without abortion would not have been found, cytological material was collected from 31 head rows in which all plants had normal pollen. These were sibs of the original head rows which had partially sterile plants. All have been checked. All had 
Table 2. Cytological configurations at meiosis in $F_{1}$ plants of intercrosses between the tester set of interchanges, and between them and a group of four others

\begin{tabular}{|c|c|c|c|c|c|c|}
\hline & $\begin{array}{c}\text { Tester inte } \\
\text { c } 1385 \\
\mathrm{a}-\mathrm{b}\end{array}$ & $\begin{array}{c}\text { change } \\
\text { c } 1358 \\
b-\mathrm{d}\end{array}$ & $\begin{array}{c}t \text {, with } \\
\text { c } 1432 \\
\text { c-e }\end{array}$ & $\begin{array}{c}\text { romoso } \\
\text { e } 1420 \\
\text { e-f }\end{array}$ & $\begin{array}{c}\text { es invo } \\
\text { e } 1405 \\
\text { c-d }\end{array}$ & $\begin{array}{l}\text { ed } \\
\text { chromosomes } \\
\text { interchanged }\end{array}$ \\
\hline \multicolumn{7}{|l|}{ Testers : } \\
\hline c $1385(a-b)$ & - & & & & & \\
\hline c $1358(b-d)$ & 96 & - & & & & \\
\hline c 1432 (c-e) & $2 \bigodot 4$ & $2 \circlearrowright 4$ & 一 & & & \\
\hline e 1420 (e-f) & $2 \bigodot 4$ & 264 & 06 & - & & \\
\hline $\mathrm{e} 1405(\mathrm{c}-\mathrm{d})$ & 204 & (e) 6 & 66 & $2(0) 4$ & - & \\
\hline \multicolumn{7}{|l|}{ Others: } \\
\hline c 1346 & 66 & 06 & 66 & 66 & - & $b+e$ \\
\hline e 1310 & 66 & 66 & 204 & $\varnothing 6$ & - & $b+f$ \\
\hline e 1462 & $\varnothing 6$ & 06 & 204 & $2 \bigodot 4$ & $2(\boldsymbol{\varphi} 4$ & $b+g$ \\
\hline erectoides -7 & $7 \mathrm{II}$ & $\varnothing 6$ & $2 \bigodot 4$ & 204 & - & $a+b$ \\
\hline
\end{tabular}

Note that to complete the identification of $\mathrm{c} 1462$, the cross with $\mathrm{c} 1405$ was needed. Without that intercross, $a-d$ or $b-g$ will fit the results.

seven pairs of chromosomes. For 15 of the lines four plants were examined, eight had three plants, five had only two, and three had only one. The numbers are adequate only to conclude that if rings without abortion exist in barley they are not as common as rings with abortion.

\section{Chromosome morphology}

During the course of this preliminary cytological study, and an earlier study, some observations on chromosome morphology were made on the Mars variety. Two chromosome pairs bear satellites, one satellite being much larger than the other as reported by Levitsky (16) and confirmed since then by $\mathrm{Tjio}$ and Hagberg (23) and others.

At diakinesis the chromosome pair with the larger satellite could be recognized in many of the cells and it was usually attached to a nucleolus. In occasional cells the pair with the smaller satellite could be recognized. In a few figures showing two nucleoli, one chromosome pair was clearly attached to the smaller nucleolus (Fig. 1) and one to the larger one. In one count in a plant of the Mars variety, of 166 sporocytes at diakinesis, 6.6 per cent of them had two nucleoli.

In the microspores of normal plants, there is a much higher percentage with two nucleoli. In two counts on microspores, 25 and 35 per cent of them had two nucleoli, usually of unequal size, the others having only one nucleolus. The evidence from both the microspores and diakinesis indicates that two chromosome pairs have a nucleolar organizer region. These are probably the two satellited chromosomes.

The association with the nucleolus at diakinesis is not as intimate as in maize. In maize the nucleolus may show a beaked appearance at the point 
of attachment to chromosome 6 , as if in the smearing process the chromosome had pulled the nucleolus with it. This extreme appearance has not been seen in barley.

The cells are smaller and the chromosomes are much thicker and longer at the pachytene and diakinesis stages than those in maize, making it more difficult to analyse the configurations. The centromere regions are difficult to distinguish with this technique. They are usually recognizable at prophase of the first microspore division.

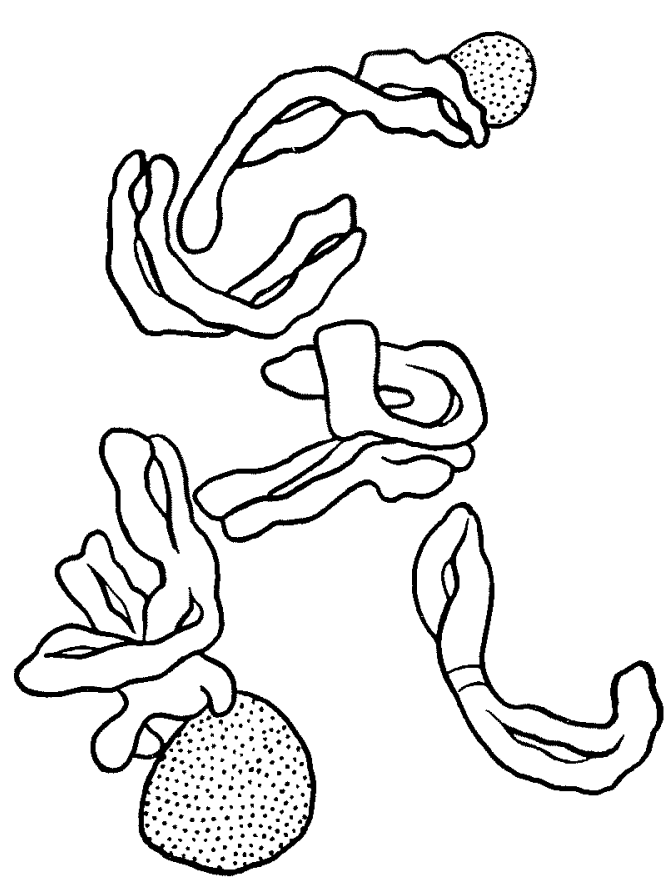

Fig. 1. Diakinesis stage of c 1376-1 with two rings of four chromosomes each and three pairs. There are two nucleoli, one associated with a chromosome pair, the other with one of the rings.

At metaphase $I$ in normal material, cells with 6 ring bivalents and one rod are frequent. Only occasionally are there two rod bivalents. At prophase of the first microspore division, only one chromosome of the seven has a short arm. It is probable, therefore, that the rod bivalent most frequently seen at metaphase $I$ is this chromosome pair. According to Hagberg and Tjio, based on root tip smears, the chromosome with the shortest arm is also the one with the small satellite. Therefore at least two of the chromosomes have a distinctive morphology recognizable at diakinesis or metaphase I. In material showing a ring or a chain of four chromosomes in the preliminary survey, incidental observations were made at diakinesis to determine if a satellite-bearing pair were in the ring, and at metaphase $I$ to determine if the rod bivalent were in the ring. In the plant having two rings of four, one ring clearly included the large satellite pair and when two nucleoli were present a few figures showed that the smaller nucleolus was associated with a chromosome pair not in either ring (Fig. 1).

In the preliminary observations, the $c 1420$ (e-f) ring was recorded as being associated with the nucleolus. After Hagberg and Tjio's report (10) that the c 1483 ring $(\mathrm{b}-\mathrm{g})$ involves the large satellite-bearing chromosome, a check of diakinesis stages of various intercrosses indicates that the original observation on c 1420 (e-f) was incorrect, and that the $g$ chromosome is the 
one bearing the large satellite, their number VI. Based on somatic chromosomes in root tips, they have numbered the chromosomes from I to VII, I being the longest. They have reported observations on c 1358 (b-d) also, from which they concluded that $d$ is their chromosome VII bearing the small satellite.

They also concluded from that study that $b$ was III, but in an earlier report (9) they had identified the chromosomes involved in the erectoides-7 interchange as $I$ and $V$. They kindly furnished seed of it, and our intercrosses (Table 2) show it to be a-b. According to a personal communication they have obtained the same result, and have concluded that the $b$ chromosome may be III or I.

The chromosomal identifications of the other interchanges will be reported later. A preliminary summary has been reported (7a).

\section{Linkage information}

Based on unpublished results by White (White and Burnham 1948) ; c 1385 (a-b) is linked with $n$ in linkage group III c 1420 (e-f) is linked with $V$ in group $I$ and $K$ in group IV c 1432 (c-e) is linked with $K$ and $B l$ in group IV c 1456 (a-e) is linked with $K$ and $B l$ in group IV ; from which it was concluded that the $e$ chromosome carries linkage group IV and that the $f$ chromosome probably carries group I. Linkage with $V$ was reported by Joachim (1947) for the " A" interchange. These linkages (excluding those with "A ") were confirmed later by data reported by Hanson and Kramer (1949) and Hanson (1950) for these same interchanges. The linkage of c 1358 (b-d) with $n$, Livers unpublished, and their linkage of c $1478(\mathrm{~b}-\mathrm{d})$ and of c 1025 (b-d) with $n$ along with the above linkage of c 1385 (a-b) with $n$ indicates that the $b$ chromosome carries group III.

By means of linkage tests with interchanges, Hanson and Kramer (11) have located the previously unlinked light-green-3 (1g3) character in linkage group IV, illustrating the use of interchanges as supplements to the genetic markers in locating new genes. This linkage tester stock had been selected at our laboratory as one of the genetic testers for linkage studies with interchanges, not only because it had gene markers known to be in several linkage groups; but also with the expectation that the unlinked $1 g-3$ might be located during the course of the linkage studies between the interchanges and the other markers in the same stock. Another example is Suneson's male sterile ( $m s$ ) first reported to be linked with interchange c 1317 (b-d) by O. Smith (21). Subsequent tests here show linkage also with c 1385 (a-b) indicating this $m s$ is probably in the $b$ chromosome (Plessers, unpublished). When the breakage points of more interchanges have been located it will be possible to select a more efficient set of interchanges for use in testing linkages. 


\section{Summary}

Thirty-four chromosomal interchanges are reported, the result of X-raying of seeds of barley. One line had two separate rings of four chromosomes each.

Pollen abortion in plants with one ring ranged in different lines from 14 to 58 per cent, the average being 28.8. The search for a line with rings but no pollen abortion was unsuccessful, however, the number of lines examined was not large.

Stocks homozygous for the interchange have been established for 28 of the 34 lines. The use of a tester set of five interchanges in identifying the chromosomes involved in new interchanges is illustrated. The chromosomes have been labeled temporarily by the letters $a$ through $g$. The testers are $a-b, b-d, c-e, e-f$, and $c-d$, the last being needed to identify only certain combinations.

Trisomics have been identified in the progeny of plants heterozygous for an interchange, the configuration at meiosis being a chain of 5 chromosomes.

The $g$ chromosome carries the large satellite, confirming the result of Hagberg and Tjio.

Linkage tests with different interchanges involving only one chromosome in common indicate that the $e$ chromosome carries linkage group IV, $f$ carries group V. b carries group III.

Their usefulness in supplementing the genetic linkage markers in tests to locate new genes is illustrated.

They will make it possible to test and establish the independence of the 7 linkage groups; to identify each linkage group with its chromosome. Studies which are in progress to determine the breakage points in the chromosomes and in the linkage groups will make them usable for special problems.

\section{Acknowledgements}

We wish to thank Drs. F.S. Warren, L. S. Negi, and Messrs. H. A. McLennan, B. Feste, E. H. Coe, J. Longwell, E. Clark, A. J. Plessers, E. Turcotte, and L. L. Inman for their contributions to various phases of the studies.

A grant from Graduate School Research Funds made possible the completion of certain phases of the work with the aid of Mrs. Amy Gage Skallerup.

\section{Literature cited}

1. Armstrong, J.M. and Huskins, C. L. 1934. Further studies on the cytology of Matthiola incana. Journ. Gen. 29: 29-50.

2. Brink, R. A. and Burnham, C. R. 1929. Inheritance of semi-sterility in maize. Amer. Nat. 63: 301-316. 
3. Burnham, C. R. 1932. An interchange in maize giving low sterility and chain configurations. Proc. Nat. Acad. of Sciences 18: 434-440.

4. - 1947. A gene for "long' chromosomes in barley. Genetics 31 (2): 212-213.

5. - 1949. Chromosome segregation in maize translocations in relation to erossing over in interstitial segments. Proc. Nat. Acad. Sciences 35: 349-356.

6. - 1950. Chromosome segregation in translocations involving chromosome 6 in maize. Genetics 35: 446-481.

7. - et al. 1951. A summary of available data on chromosome translocations (based on data from W. D. Hanson and H. H. Kramer; M. Waddoups and R. W. Woodward; O. Smith and O.J. Webster; and F.H. White, R. Livers and C.R. Burnham). Presented at Barley Workers Conference (mimeographed), Madison, Wise. January.

7a. - White, F.H. and Livers. R. 1953, Chromosome translocations in barley. Proe. Ninth Internat. Congress of Genetics (Abstract). Bellagio, Italy.

8. Das, K. 1951. Cytogenetic studies of partial sterility in barley. Ph. D. thesis University of Minnesota.

9. Hagberg, A. and Tjio. J. H. 1950. Cytological localization of the translocation point for the barley mutant erectoides 7. Hereditas 36: 487-491.

10. - and Tjio. J.H. 1952. Cytological studies on some homozygous transloeations in barley. Anales Estac. Exper. Aula Dei, 2: 215-223.

11. Hanson, W.D. and Kramer. H. H., 1949. The genetic analysis of two chromosome interehanges in barley from $F_{2}$ data. Genetics 34: 687-700.

12. - 1950. The analysis and interpretation of genetic data involving translocations obtained from $F_{z}$ and $F_{3}$ generations in barley. Ph. D. thesis, Purdue University.

13. - and Kramer, H.H. 1950. The determination of linkage intensities from $F_{2}$, and $\mathrm{F}_{3}$ genetic data involving chromosomal interchanges in barley. Genetics 35: $559-569$.

14. Joachim, G.S. 1947. The product method of calculating linkage from $\mathrm{F}_{2}$ data involving semisterility and its application to a barley translocation. Genetics 32: $580-591$.

15. Lesley, M. Mann and Frost, H. B. 1927. Mendelian inheritance of chromosome shape in Matthiola. Genetics 12: 449-160.

16. Levitsky, G. A. 1931. The morphology of the chromosomes. Trudy Prikl. Bot., Genet. i. Selek. 27: 19-173.

17. MeLennan, H.A. 1947. Cytogenetic studies of a strain of barley with long chromosomes. M.S. thesis, University of Minnesota.

18. Robertson, D.W., Wiebe G. A. and Immer. F.R. 1941. A summary of linkage studies in barley. Jour. Am. Soc. Agron. 33: 47-64.

19. - Wiebe, G. A. and Shands, R. G. 1947. A summary of linkage studies in barley: supplement 1: 1940-1946. Jour. Amer. Soc. Agron. 39: 464-473.

20. Smith. L. 1941. An inversion, a reciprocal translocation, trisomics and tetraploids in barley. J. Agric. Res. 63: 741-750.

20a. - 1951. Cytology and geneties of barley. Bot. Rev. 17: 1-51, 133-202, 285-355.

21. Smith, Oliver E. (and O.J. Webster). A cytogenetic study of two translocations in barley. 1949. M. S. thesis, University of Nebraska.

22. Thompson, W. P. and Thompson, M. G. 1937. Cytologia, Fujii Jubilee Volume, Pt. 1: $336-342$.

23. Tjio, J.H. and Hagberg, A. 1951. Cytological studies on some X-ray mutants of barley. Anales Estac. Exper. Aula Dei. 2: 149-167.

24. Waddoups, Horace Mann (and R. W. Woodward). 1949. A study of semisterility and its linkage relationships in translocation stocks of barley. Master of Science thesis in Plant Breeding, Utah State Agricultural College. 
25. White, F.H. and Burnham, C. R. 1948. Chromosome translocation in barley. Paper presented at American Society of Agronomy meetings, Fort Collins, Colo. August. (abstract).

26. Yamashita, K. 1951. Studies on X-ray induced reciprocal translocations in Einkorn wheats III. A newly synthesized ring of 14 chromosomes in a complex heterozygote, Aegilopoides monococeum. Cytologia 16: 164-176. 